\title{
Control in vitro de Lasiodiplodia theobromae (Pat.) Griff. \& Maubl y L. citricola Abdollahz aislados de lima Persa (Citrus latifolia Tanaka) en Morelos, México
}

\author{
In vitro control of Lasiodiplodia theobromae (Pat.) Griff. \& Maubl and L. citricola Abdollahz isolated \\ from Persian lime (Citrus latifolia Tanaka) in Morelos, Mexico \\ Mairel Valle-de la Paz ${ }^{1}$, Dagoberto Guillén-Sánchez ${ }^{*}$, Irán Alia-Tejacal ${ }^{1}$, Víctor López-Martínez ${ }^{1}$, \\ Porfirio Juárez-López ${ }^{1}$, Edgar Martínez-Fernández², Marianguadalupe Hernández-Arenas ${ }^{3}$ y Rafael \\ Ariza-Flores ${ }^{4}$
}

\begin{abstract}
RESUMEN
El objetivo del presente estudio fue evaluar in vitro nueve fungicidas para inhibir el crecimiento micelial de Lasiodiplodia theobromae y L. citricola. Las cepas fueron aisladas de muestras colectadas de árboles de lima persa de los municipios de Tlaltizapán y Jantetelco, Morelos, México. Se evaluaron tres dosis distintas de ziram, carbendazim, thiram, tiofanato metílico, tiabendazol, cyprodinil+fludioxonil, iprodiona, Trichoderma harzianum, benomilo y un testigo absoluto sin fungicida; diluidos en $100 \mathrm{~mL}$ medio de PDA. El diseño experimental fue completamente al azar, utilizando un ANOVA simple con la prueba Kruskal-Wallis $(\alpha \leq 0.05)$. la evaluación de la efectividad fue analizada cada 24 h. Lasiodiplodia theobromae fue sensible a todas las dosis de fungicidas estudiadas, sin crecimiento micelial. Lasiodiplodia citricola registró crecimiento micelial en cyprodinil+fludioxonil, a partir de las 48 h después de la siembra, alcanzando un diámetro máximo de $2.0 \mathrm{~cm}$, en comparación con el testigo absoluto, quien llenó por completo la caja a las $96 \mathrm{~h}$.
\end{abstract}

PALABRAS CLAVE

Efectividad biológica, muerte regresiva, fungicidas, Trichoderma harzianum.

\section{ABSTRACT}

The objective of the present study was to evaluate in vitro nine fungicides to inhibit the mycelial growth of Lasiodiplodia theobromae and L. citricola. The strains were isolated from samples collected from Persian lime trees from the municipalities of Tlaltizapan and Jantetelco, Mor. He treatments were evaluated every $24 \mathrm{~h}$ with three different doses with five repetitions each and its control, the treatments: Ziram, Carbendazim, Thiram, Methyl thiophanate, Thiabendazole, Cyprodinil + fludioxonil, Iprodione, Trichoderma harzianum, Benomyl and a treatment without fungicide as reference, diluted in 100 mL of PDA. Experimental design was completely randomized, using a simple ANOVA with the Kruskal-Wallis test, $\alpha \leq 0.05$. Lasiodiplodia theobromae was sensitive to all doses of fungicides with $0.0 \mathrm{~cm}$ diameter of mycelial growth. Compared with the reference treatment who filled the box at $120 \mathrm{~h}$. While Lasiodiplodia citricola had mycelial growth in the treatments of Cyprodinil + fludioxonil, starting $48 \mathrm{~h}$ after sowing, reaching a maximum diameter of $2.0 \mathrm{~cm}$ while the reference treatment completely filled the box at $96 \mathrm{~h}$, being statistically different from the other treatments.

KEYWORDS

Biological effectiveness, dieback, fungicides, Trichoderma harzianum.

\footnotetext{
${ }^{1}$ Posgrado en Ciencias Agropecuarias y Desarrollo Rural, Facultad de Ciencias Agropecuarias Universidad Autónoma del Estado de Morelos (UAEM), Av. Universidad 1001, Col. Chamilpa, 62209, Cuernavaca, Morelos, México.

${ }^{2}$ Centro de Investigaciones Biológicas (UAEM).

${ }^{3}$ INIFAP Campo Experimental Zacatepec, Carretera Zacatepec-Galeana Km 0.5, 62780, Zacatepec, Morelos, México.

${ }^{4}$ INIFAP Campo Experimental Iguala, Carretera Iguala-Tuxpan Km 2.5, Tuxpan, 40000, Iguala de la Independencia, Guerrero, México.

*Autor para correspondencia: dagoguillen@yahoo.com
}

Fecha de recepción: 27 de noviembre de 2018.

Fecha de aceptación: 25 de febrero de 2019.

Disponible en línea: 28 de julio de 2019. 


\section{INTRODUCCIÓN}

La producción de cítricos en México, en especial de lima persa (Citrus latifolia Tanaka), se ha orientado a la producción para el consumo en fresco, y hoy en día es el exportador líder de esta fruta a escala mundial (Schwentesius y Gómez 2005). En 2017, la producción nacional fue de 6.9 millones de $t$ (Varela 2019), con un valor de la producción de 16,781 millones de pesos; para el mismo año, la superficie sembrada de cítricos fue de 533,749 ha, de las cuales 180,252 fueron de limón, 17,887 de toronja y 335,610 de naranja dulce (SIAP 2016).

Los productores mayores de lima Persa son Veracruz (717,014 t), Michoacán (619,612 t) y Oaxaca $(263,448$ t), mientras que Morelos ocupa el décimosegundo lugar, con una producción de 3,880 t. En 2010, Morelos tenía huertas de cítricos establecidas en 28 de los 33 municipios, abarcando una superficie de 1,000 ha; actualmente cuenta con 612 ha sembradas en 17 municipios, entre las cuales destacan la lima Persa y la naranja valencia, con 32 y $66 \%$, respectivamente. Ambas especies son las de mayor importancia económica, con rendimientos de 12.11 y 7.76 t ha- 1 , respectivamente (CESVMOR 2010; SIAP 2016).

Los productores asociaban al patógeno Phytophthora parasitica con los síntomas de gomosis y muerte descendente en cítricos, debido a que varios autores habían reportado este patógeno como el agente causal. Fawcett (1936) lo encontró en Argentina atacando cítricos; Frezzi (1950) reportó varias especies de cítricos como hospedantes de este patógeno, incluidos, por orden cronológico: Citrus sinensis (L.) Osbeck (1937), C. aurantiifolia (Christm.) Swingle (1938), C. nobilis Lour (1939), C. x limonia Osbeck (1939). En México, Vidales (1982) reportó por primera vez esta especie como la causante de la gomosis en la región citrícola de Colima. Acosta-Pérez et al. (2012) mencionan que la gomosis o pudrición del pie de los cítricos ha sido una de las enfermedades más importantes, ya que anualmente produce la muerte de muchos árboles de cítricos de pie franco e injertados. Estos antecedentes fueron considerados en Morelos para recomendar a los productores de cítricos la aplicación de Fosetil Aluminio y Metalaxil en el control de esta enfermedad; sin embargo, los resultados no fueron exitosos.

Valle (2018) llevó a cabo la identificación y el reporte de otras especies que ocasionaban la gomosis y muerte descendente en árboles de lima Persa en Morelos: tales como Lasiodiplodia citricola, L. pseudotheobromae y L. theobromae (Botryosphaeriales: Dothideomycetes: Ascomycota: Botryosphaeriaceae), especies cosmopolitas que se encuentran en una diversidad de hospedantes monocotiledóneas, dicotiledóneas y gimnospermas (Abdollahzadeh et al. 2010).

La muerte descendente es una enfermedad de importancia en lima Persa en Morelos, con incidencia de 40 a 70\% (Valle-de la Paz et al. 2015). Sin embargo, se carece de información actual sobre la eficacia de las moléculas fungicidas disponibles; por ello, el objetivo del presente trabajo fue evaluar el efecto in vitro de diversos fungicidas químicos y un producto biológico en la inhibición micelial de Lasiodiplodia theobromae y Lasiodiplodia citricola.

\section{MATERIALES Y MÉTODOS}

Para el presente estudio, se usaron dos cepas identificadas molecularmente con número de acceso GenBank Lasiodiplodia theobromae KY284587 y Lasiodiplodia citricola KY271187 (Valle 2018; Valle et al. 2019), las cuales fueron aisladas de tallo principal de árboles de lima Persa de los municipios de Tlaltizapán y Jantetelco, Morelos, México (cuadro 1). Estas cepas indujeron la producción de goma y muerte descendente en árboles de 8 años. El trabajo fue efectuado en el Laboratorio de Fitopatología de la Escuela de Estudios Superiores de Xalostoc, de la Universidad Autónoma del Estado de Morelos.

El método utilizado fue por envenenamiento del medio: los fungicidas previamente se diluían en 5 $\mathrm{mL}$ de agua destilada desinfectada, posteriormente se mezclaban en $95 \mathrm{~mL}$ de medio de cultivo papa-dextrosa-agar (PDA) (BD Bioxon $\left.{ }^{\circledR}\right)$, previamente esterilizado en autoclave; como control se utilizó PDA sin fungicida. Se evaluaron tres dosis diferentes de cada fungicida seleccionado; la dosis alta fue la recomendada en la etiqueta de cada producto, una dosis media y una baja de cada tratamiento evaluado (cuadro 2). Se vertían aproximadamente $20 \mathrm{~mL}$ de medio con fungicida en cada caja de Petri de $90 \times 15$ $\mathrm{mm}$. En el centro de cada caja se colocó un disco de 0.5 $\mathrm{cm}$ de diámetro con medio de cultivo y micelio de tres días de edad, previamente purificados por el método de punta de hifa. Las cajas se incubaron a $28^{\circ} \mathrm{C}$.

La variable de estudio fue el diámetro de las colonias, el cual se evaluó cada 24 h con un vernier digital, hasta que el tratamiento control llenó la caja por completo. Se usó un diseño experimental completamente al azar, con 28 tratamientos (tres dosis de nueve fungicidas y un testigo sin fungicida) y cinco repeticiones. Los datos fueron sometidos a un análisis de varianza ANOVA simple, determinando comparación de medias, con la prueba Kruskal-Wallis, $\alpha \leq 0.05$. 
Cuadro 1. Localización de huertos donde se obtuvieron los aislamientos de las cepas de Lasiodiplodia theobromae y L. citricola empleados en la evaluación in vitro

\begin{tabular}{|c|c|c|c|c|c|}
\hline Huerto & Municipio & Localidad & $\begin{array}{l}\text { Coordenadas } \\
\text { geográficas }\end{array}$ & $\begin{array}{c}\text { Altitud } \\
\text { sobre el } \\
\text { nivel del mar }\end{array}$ & Patógeno \\
\hline 1 & Tlaltizapán & San Rafael & $\begin{array}{l}18.65262 \\
-99.03149\end{array}$ & 1,032 & L. theobromae \\
\hline 2 & Tlaltizapán & San Rafael & $\begin{array}{l}18.63525 \\
-99.00971\end{array}$ & 1,003 & L. theobromae \\
\hline 36 & Jantetelco & Amayuca & $\begin{array}{l}\text { 18.71317, } \\
-98.79353\end{array}$ & 1,425 & L. citricola \\
\hline 37 & Jantetelco & Amayuca & $\begin{array}{l}18.73256 \\
-98.78864\end{array}$ & 1,474 & L. citricola \\
\hline
\end{tabular}

Cuadro 2. Fungicidas evaluados in vitro sobre Lasiodiplodia theobromae y Lasiodiplodia citricola, aislados de lima Persa (Citrus latifolia Tanaka) con síntomas de gomosis y muerte descendente de Morelos, México

\begin{tabular}{|c|c|c|c|c|c|c|c|}
\hline \multirow[t]{2}{*}{$\begin{array}{c}\text { Grupo } \\
\text { químico }\end{array}$} & \multirow[t]{2}{*}{$\begin{array}{l}\text { Ingrediente } \\
\text { activo (i. a.) }\end{array}$} & \multirow[t]{2}{*}{$\begin{array}{l}\text { Modo de } \\
\text { acción }\end{array}$} & \multirow[t]{2}{*}{ \% i.a. } & \multirow[t]{2}{*}{ Formulación } & \multicolumn{3}{|c|}{$\begin{array}{l}\text { Tratamientos } \\
\text { Dosis } \\
\text { (g/100 mL agua) }\end{array}$} \\
\hline & & & & & Alta & Media & Baja \\
\hline Ditiocarbamato & Ziram & C & 76 & GD & 0.4 & 0.35 & 0.3 \\
\hline Benzimidazol & Carbendazim & S & 50 & WG & 0.19 & 0.15 & 0.11 \\
\hline Ditiocarbamato & Thiram & C & 80 & GD & 0.19 & 0.15 & 0.11 \\
\hline Benzimidazol & $\begin{array}{c}\text { Tiofanato } \\
\text { metílico }\end{array}$ & S & 70 & $\mathrm{PH}$ & 0.1 & 0.08 & 0.06 \\
\hline Benzimidazol & Tiabendazol & S & 60 & WP & 0.29 & 0.24 & 0.19 \\
\hline $\begin{array}{c}\text { Anilino- } \\
\text { pirimidinas/ } \\
\text { Fenilpirroles }\end{array}$ & $\begin{array}{l}\text { Cyprodinil + } \\
\text { fludioxonil }\end{array}$ & Cys & 37.5 & WP & 0.39 & 0.31 & 0.23 \\
\hline Dicarboximidas & Iprodiona & C & 50 & $\mathrm{PH}$ & 0.58 & 0.39 & 0.19 \\
\hline Biofungicida & $\begin{array}{l}\text { Trichoderma } \\
\text { harzianum }\end{array}$ & $\mathrm{C}$ & $1 \times 108 \mathrm{UFC} / \mathrm{g}$ & $\mathrm{PH}$ & 0.58 & 0.39 & 0.19 \\
\hline Benzimidazol & Benomilo & S & 50 & $\mathrm{PH}$ & 0.09 & 0.075 & 0.06 \\
\hline ----- & Testigo absoluto & --.-- & -ב-ב-ב-ב- & & 0 & 0 & 0 \\
\hline
\end{tabular}

$\mathrm{S}=$ fungicida sistémico, $\mathrm{C}=$ fungicida de contacto, $\mathrm{GD}=$ gránulos dispersables, $\mathrm{WG}=$ gránulos dispersables, $\mathrm{PH}=$ polvo humectable, $\mathrm{WP}=$ polvo mojable.

\section{RESUlTADOS Y DISCUSIÓN}

Lasiodiplodia theobromae fue altamente sensible a todas las dosis de los fungicidas evaluados, debido a que no presentó crecimiento miceliar; en comparación, el testigo absoluto llenó la caja Petri a las $120 \mathrm{~h}$ después de la siembra de los patógenos (cuadro 3). De manera similar, Lasiodiplodia citricola fue sensible en gran medida a los fungicidas Ziram, Carbendazim,
Thiram, Tiofanato metílico, Tiabendazol, Iprodiona, Trichoderma harzianum y Benomilo, quienes inhibieron completamente el crecimiento micelial. El tratamiento con Cyprodinil + fludioxonil presentó crecimiento a partir de las $48 \mathrm{~h}$ después de la siembra, alcanzando una media de diámetro de $1.94 \mathrm{~cm}$ en la dosis alta, $1.96 \mathrm{~cm}$ en la dosis media y $2.0 \mathrm{~cm}$ en la dosis baja (cuadro 4), mientras que el testigo absoluto llenó por completo la caja a las $96 \mathrm{~h}$. 
Cuadro 3. Efectividad de fungicidas in vitro sobre Lasiodiplodia theobromae, aislados de lima Persa (Citrus latifolia Tanaka) con síntomas de gomosis y muerte descendente de Morelos, México

\begin{tabular}{|c|c|c|c|c|c|c|c|c|c|}
\hline \multirow[t]{3}{*}{ Tratamiento } & \multirow{3}{*}{$\begin{array}{l}\text { Ingrediente } \\
\text { activo (i.a.) }\end{array}$} & \multirow[t]{3}{*}{ Dosis } & \multirow{2}{*}{\multicolumn{7}{|c|}{$\begin{array}{c}\text { Lasiodiplodia theobromae } \\
\text { Número de evaluación }\end{array}$}} \\
\hline & & & & & & & & & \\
\hline & & & 1 & 2 & 3 & 4 & 5 & 6 & 7 \\
\hline \multirow[t]{3}{*}{1} & \multirow[t]{3}{*}{ Ziram } & Alta & $0.0^{\mathrm{b}}$ & $0.0 \mathrm{~b}$ & $0.0 \mathrm{~b}$ & $0.0^{\mathrm{b}}$ & $0.0^{\mathrm{b}}$ & $0.0^{\mathrm{b}}$ & $0.0^{\mathrm{b}}$ \\
\hline & & Media & $0.0^{\mathrm{b}}$ & $0.0 \mathrm{~b}$ & $0.0 \mathrm{~b}$ & $0.0^{\mathrm{b}}$ & $0.0^{\mathrm{b}}$ & $0.0^{\mathrm{b}}$ & $0.0^{\mathrm{b}}$ \\
\hline & & Baja & $0.0^{\mathrm{b}}$ & $0.0 \mathrm{~b}$ & $0.0 \mathrm{~b}$ & $0.0^{\mathrm{b}}$ & $0.0^{\mathrm{b}}$ & $0.0^{\mathrm{b}}$ & $0.0^{\mathrm{b}}$ \\
\hline \multirow[t]{3}{*}{2} & \multirow[t]{3}{*}{ Carbendazim } & Alta & $0.0^{\mathrm{b}}$ & $0.0^{\mathrm{b}}$ & $0.0^{\mathrm{b}}$ & $0.0^{\mathrm{b}}$ & $0.0^{\mathrm{b}}$ & $0.0^{\mathrm{b}}$ & $0.0^{\mathrm{b}}$ \\
\hline & & Media & $0.0^{\mathrm{b}}$ & $0.0^{\mathrm{b}}$ & $0.0^{\mathrm{b}}$ & $0.0^{\mathrm{b}}$ & $0.0^{\mathrm{b}}$ & $0.0^{\mathrm{b}}$ & $0.0^{\mathrm{b}}$ \\
\hline & & Baja & $0.0^{\mathrm{b}}$ & $0.0^{\mathrm{b}}$ & $0.0^{\mathrm{b}}$ & $0.0^{\mathrm{b}}$ & $0.0^{\mathrm{b}}$ & $0.0^{\mathrm{b}}$ & $0.0^{\mathrm{b}}$ \\
\hline \multirow[t]{3}{*}{3} & \multirow[t]{3}{*}{ Thiram } & Alta & $0.0^{\mathrm{b}}$ & $0.0^{\mathrm{b}}$ & $0.0^{\mathrm{b}}$ & $0.0^{\mathrm{b}}$ & $0.0^{\mathrm{b}}$ & $0.0^{\mathrm{b}}$ & $0.0^{\mathrm{b}}$ \\
\hline & & Media & $0.0^{\mathrm{b}}$ & $0.0^{\mathrm{b}}$ & $0.0^{\mathrm{b}}$ & $0.0^{\mathrm{b}}$ & $0.0^{\mathrm{b}}$ & $0.0^{\mathrm{b}}$ & $0.0^{\mathrm{b}}$ \\
\hline & & Baja & $0.0^{\mathrm{b}}$ & $0.0^{\mathrm{b}}$ & $0.0^{\mathrm{b}}$ & $0.0^{\mathrm{b}}$ & $0.0^{\mathrm{b}}$ & $0.0^{\mathrm{b}}$ & $0.0^{\mathrm{b}}$ \\
\hline \multirow[t]{3}{*}{4} & \multirow{3}{*}{$\begin{array}{l}\text { Tiofanato } \\
\text { metílico }\end{array}$} & Alta & $0.0^{\mathrm{b}}$ & $0.0^{\mathrm{b}}$ & $0.0^{\mathrm{b}}$ & $0.0^{\mathrm{b}}$ & $0.0^{\mathrm{b}}$ & $0.0^{\mathrm{b}}$ & $0.0^{\mathrm{b}}$ \\
\hline & & Media & $0.0^{\mathrm{b}}$ & $0.0^{\mathrm{b}}$ & $0.0^{\mathrm{b}}$ & $0.0^{\mathrm{b}}$ & $0.0^{\mathrm{b}}$ & $0.0^{\mathrm{b}}$ & $0.0^{\mathrm{b}}$ \\
\hline & & Baja & $0.0^{\mathrm{b}}$ & $0.0^{\mathrm{b}}$ & $0.0^{\mathrm{b}}$ & $0.0^{\mathrm{b}}$ & $0.0^{\mathrm{b}}$ & $0.0^{\mathrm{b}}$ & $0.0^{\mathrm{b}}$ \\
\hline \multirow[t]{3}{*}{5} & \multirow[t]{3}{*}{ Tiabendazol } & Alta & $0.0^{\mathrm{b}}$ & $0.0^{\mathrm{b}}$ & $0.0^{\mathrm{b}}$ & $0.0^{\mathrm{b}}$ & $0.0^{\mathrm{b}}$ & $0.0^{\mathrm{b}}$ & $0.0^{\mathrm{b}}$ \\
\hline & & Media & $0.0^{\mathrm{b}}$ & $0.0^{\mathrm{b}}$ & $0.0^{\mathrm{b}}$ & $0.0^{\mathrm{b}}$ & $0.0^{\mathrm{b}}$ & $0.0^{\mathrm{b}}$ & $0.0^{\mathrm{b}}$ \\
\hline & & Baja & $0.0^{\mathrm{b}}$ & $0.0^{\mathrm{b}}$ & $0.0^{\mathrm{b}}$ & $0.0^{\mathrm{b}}$ & $0.0^{\mathrm{b}}$ & $0.0^{\mathrm{b}}$ & $0.0^{\mathrm{b}}$ \\
\hline \multirow[t]{3}{*}{6} & \multirow{3}{*}{$\begin{array}{l}\text { Cyprodinil + } \\
\text { fludioxonil }\end{array}$} & Alta & $0.0^{\mathrm{b}}$ & $0.0^{\mathrm{b}}$ & $0.0^{\mathrm{b}}$ & $0.0^{\mathrm{b}}$ & $0.0^{\mathrm{b}}$ & $0.0^{\mathrm{b}}$ & $0.0^{\mathrm{b}}$ \\
\hline & & Media & $0.0^{\mathrm{b}}$ & $0.0^{\mathrm{b}}$ & $0.0^{\mathrm{b}}$ & $0.0^{\mathrm{b}}$ & $0.0^{\mathrm{b}}$ & $0.0^{\mathrm{b}}$ & $0.0^{\mathrm{b}}$ \\
\hline & & Baja & $0.0^{\mathrm{b}}$ & $0.0^{\mathrm{b}}$ & $0.0^{\mathrm{b}}$ & $0.0^{\mathrm{b}}$ & $0.0^{\mathrm{b}}$ & $0.0^{\mathrm{b}}$ & $0.0^{\mathrm{b}}$ \\
\hline \multirow[t]{3}{*}{7} & \multirow[t]{3}{*}{ Iprodiona } & Alta & $0.0^{\mathrm{b}}$ & $0.0^{\mathrm{b}}$ & $0.0^{\mathrm{b}}$ & $0.0^{\mathrm{b}}$ & $0.0^{\mathrm{b}}$ & $0.0^{\mathrm{b}}$ & $0.0^{\mathrm{b}}$ \\
\hline & & Media & $0.0^{\mathrm{b}}$ & $0.0^{\mathrm{b}}$ & $0.0^{\mathrm{b}}$ & $0.0^{\mathrm{b}}$ & $0.0^{\mathrm{b}}$ & $0.0^{\mathrm{b}}$ & $0.0^{\mathrm{b}}$ \\
\hline & & Baja & $0.0^{\mathrm{b}}$ & $0.0^{\mathrm{b}}$ & $0.0^{\mathrm{b}}$ & $0.0^{\mathrm{b}}$ & $0.0^{\mathrm{b}}$ & $0.0^{\mathrm{b}}$ & $0.0^{\mathrm{b}}$ \\
\hline \multirow[t]{3}{*}{8} & \multirow{3}{*}{$\begin{array}{l}\text { Trichoderma } \\
\text { harzianum }\end{array}$} & Alta & $0.0^{\mathrm{b}}$ & $0.0^{\mathrm{b}}$ & $0.0^{\mathrm{b}}$ & $0.0^{\mathrm{b}}$ & $0.0^{\mathrm{b}}$ & $0.0^{\mathrm{b}}$ & $0.0^{\mathrm{b}}$ \\
\hline & & Media & $0.0^{\mathrm{b}}$ & $0.0^{\mathrm{b}}$ & $0.0^{\mathrm{b}}$ & $0.0^{\mathrm{b}}$ & $0.0^{\mathrm{b}}$ & $0.0^{\mathrm{b}}$ & $0.0^{\mathrm{b}}$ \\
\hline & & Baja & $0.0^{\mathrm{b}}$ & $0.0^{\mathrm{b}}$ & $0.0^{\mathrm{b}}$ & $0.0^{\mathrm{b}}$ & $0.0^{\mathrm{b}}$ & $0.0^{\mathrm{b}}$ & $0.0^{\mathrm{b}}$ \\
\hline \multirow[t]{3}{*}{9} & \multirow[t]{3}{*}{ Benomilo } & Alta & $0.0^{\mathrm{b}}$ & $0.0^{\mathrm{b}}$ & $0.0^{\mathrm{b}}$ & $0.0^{\mathrm{b}}$ & $0.0^{\mathrm{b}}$ & $0.0^{\mathrm{b}}$ & $0.0^{\mathrm{b}}$ \\
\hline & & Media & $0.0^{\mathrm{b}}$ & $0.0^{\mathrm{b}}$ & $0.0^{\mathrm{b}}$ & $0.0^{\mathrm{b}}$ & $0.0^{\mathrm{b}}$ & $0.0^{\mathrm{b}}$ & $0.0^{\mathrm{b}}$ \\
\hline & & Baja & $0.0^{\mathrm{b}}$ & $0.0^{\mathrm{b}}$ & $0.0^{\mathrm{b}}$ & $0.0^{\mathrm{b}}$ & $0.0^{\mathrm{b}}$ & $0.0^{\mathrm{b}}$ & $0.0^{\mathrm{b}}$ \\
\hline 10 & $\begin{array}{l}\text { Testigo } \\
\text { absoluto }\end{array}$ & ----- & $3.5^{\mathrm{a}}$ & $4.8^{\mathrm{a}}$ & $6.0^{\mathrm{a}}$ & $6.76^{\mathrm{a}}$ & $7.34^{\mathrm{a}}$ & $7.76^{\mathrm{a}}$ & $8.0^{\mathrm{a}}$ \\
\hline
\end{tabular}

* En columnas para cada factor principal letras similares indican que los valores son estadísticamente iguales (Tukey, $\alpha=0.05$ ). 
Cuadro 4. Efectividad de fungicidas in vitro sobre Lasiodiplodia citricola, aislados de lima Persa (Citrus latifolia Tanaka) con síntomas de gomosis y muerte descendente de Morelos, México

\begin{tabular}{|c|c|c|c|c|c|c|}
\hline \multirow[t]{3}{*}{ Tratamiento } & \multirow{3}{*}{$\begin{array}{l}\text { Ingrediente } \\
\text { activo (i. a.) }\end{array}$} & \multirow[t]{3}{*}{ Dosis } & \multirow{2}{*}{\multicolumn{4}{|c|}{$\begin{array}{l}\text { Lasiodiplodia citricola } \\
\text { Número de evaluación }\end{array}$}} \\
\hline & & & & & & \\
\hline & & & 1 & 2 & 3 & 4 \\
\hline \multirow[t]{3}{*}{1} & Ziram & Alta & $0.0^{\mathrm{b}}$ & $0.0^{\mathrm{d}}$ & $0.0^{c}$ & $0.0^{c}$ \\
\hline & & Media & $0.0^{\mathrm{b}}$ & $0.0^{\mathrm{d}}$ & $0.0^{c}$ & $0.0^{c}$ \\
\hline & & Baja & $0.0^{\mathrm{b}}$ & $0.0^{\mathrm{d}}$ & $0.0^{c}$ & $0.0^{c}$ \\
\hline \multirow[t]{3}{*}{2} & Carbendazim & Alta & $0.0^{\mathrm{b}}$ & $0.0^{\mathrm{d}}$ & $0.0^{c}$ & $0.0^{c}$ \\
\hline & & Media & $0.0^{\mathrm{b}}$ & $0.0^{\mathrm{d}}$ & $0.0^{c}$ & $0.0^{c}$ \\
\hline & & Baja & $0.0^{\mathrm{b}}$ & $0.0^{\mathrm{d}}$ & $0.0^{c}$ & $0.0^{c}$ \\
\hline \multirow[t]{3}{*}{3} & Thiram & Alta & $0.0^{\mathrm{b}}$ & $0.0^{\mathrm{d}}$ & $0.0^{c}$ & $0.0^{c}$ \\
\hline & & Media & $0.0^{\mathrm{b}}$ & $0.0^{\mathrm{d}}$ & $0.0^{c}$ & $0.0^{c}$ \\
\hline & & Baja & $0.0^{\mathrm{b}}$ & $0.0^{\mathrm{d}}$ & $0.0^{c}$ & $0.0^{c}$ \\
\hline \multirow[t]{3}{*}{4} & $\begin{array}{l}\text { Tiofanato } \\
\text { metílico }\end{array}$ & Alta & $0.0^{\mathrm{b}}$ & $0.0^{\mathrm{d}}$ & $0.0^{c}$ & $0.0^{c}$ \\
\hline & & Media & $0.0^{\mathrm{b}}$ & $0.0^{\mathrm{d}}$ & $0.0^{c}$ & $0.0^{c}$ \\
\hline & & Baja & $0.0^{\mathrm{b}}$ & $0.0^{\mathrm{d}}$ & $0.0^{c}$ & $0.0^{c}$ \\
\hline \multirow[t]{3}{*}{5} & Tiabendazol & Alta & $0.0^{\mathrm{b}}$ & $0.0^{\mathrm{d}}$ & $0.0^{c}$ & $0.0^{c}$ \\
\hline & & Media & $0.0^{\mathrm{b}}$ & $0.0^{\mathrm{d}}$ & $0.0^{c}$ & $0.0^{c}$ \\
\hline & & Baja & $0.0^{\mathrm{b}}$ & $0.0^{\mathrm{d}}$ & $0.0^{c}$ & $0.0^{c}$ \\
\hline \multirow[t]{3}{*}{6} & $\begin{array}{l}\text { Cyprodinil + } \\
\text { fludioxonil }\end{array}$ & Alta & $0.0^{\mathrm{b}}$ & $0.70^{\mathrm{cb}}$ & $1.58^{\mathrm{b}}$ & $1.94^{\mathrm{b}}$ \\
\hline & & Media & $0.0^{\mathrm{b}}$ & $0.68^{c}$ & $1.52^{\mathrm{b}}$ & $1.96^{\mathrm{b}}$ \\
\hline & & Baja & $0.0^{\mathrm{b}}$ & $0.94^{\mathrm{b}}$ & $1.50^{\mathrm{b}}$ & $2.0^{\mathrm{b}}$ \\
\hline \multirow[t]{3}{*}{7} & Iprodiona & Alta & $0.0^{\mathrm{b}}$ & $0.0^{\mathrm{d}}$ & $0.0^{c}$ & $0.0^{c}$ \\
\hline & & Media & $0.0^{\mathrm{b}}$ & $0.0^{\mathrm{d}}$ & $0.0^{c}$ & $0.0^{c}$ \\
\hline & & Baja & $0.0^{\mathrm{b}}$ & $0.0^{\mathrm{d}}$ & $0.0^{c}$ & $0.0^{c}$ \\
\hline \multirow[t]{3}{*}{8} & $\begin{array}{l}\text { Trichoderma } \\
\text { harzianum }\end{array}$ & Alta & $0.0^{\mathrm{b}}$ & $0.0^{\mathrm{d}}$ & $0.0^{c}$ & $0.0^{c}$ \\
\hline & & Media & $0.0^{\mathrm{b}}$ & $0.0^{\mathrm{d}}$ & $0.0^{c}$ & $0.0^{c}$ \\
\hline & & Baja & $0.0^{\mathrm{b}}$ & $0.0^{\mathrm{d}}$ & $0.0^{c}$ & $0.0^{c}$ \\
\hline \multirow[t]{3}{*}{9} & Benomilo & Alta & $0.0^{\mathrm{b}}$ & $0.0^{\mathrm{d}}$ & $0.0^{c}$ & $0.0^{c}$ \\
\hline & & Media & $0.0^{\mathrm{b}}$ & $0.0^{\mathrm{d}}$ & $0.0^{c}$ & $0.0^{c}$ \\
\hline & & Baja & $0.0^{\mathrm{b}}$ & $0.0^{\mathrm{d}}$ & $0.0^{c}$ & $0.0^{c}$ \\
\hline 10 & $\begin{array}{c}\text { Testigo } \\
\text { absoluto }\end{array}$ & ---- & $3.5^{\mathrm{a}}$ & $4.64^{\mathrm{a}}$ & $7.1^{\mathrm{a}}$ & $8.0^{\mathrm{a}}$ \\
\hline
\end{tabular}

* En columnas para cada factor principal letras similares indican que los valores son estadísticamente iguales (Tukey, $\alpha=0.05$ ).

Los resultados en el presente estudio concuerdan con lo reportado por otros investigadores. Tovar et al. (2013) evaluaron in vitro la mezcla de cyprodinil + fludioxinil sobre L. theobromae, causante de la muerte 
descendente en varetas de mamey, y obtuvieron EC50 $=0.0043 \mu \mathrm{g} \mathrm{mL}-1$. Nath et al. (2014) evaluaron in vitro la efectividad de mancozeb a 2,500 ppm contra $L$. theobromae, causante de la podredumbre de los frutos del banano después de la cosecha en la región del sur de Gujarat, India. Este fungicida inhibió completamente el crecimiento micelial de L. theobromae, mientras que T. harzianum inhibió el crecimiento micelial de L. theobromae en $47.5 \%$, mostrando antagonismo moderado. Banik et al. (1998) reportaron la inhibición del crecimiento micelial de Botryodiplodia theobromae, agente causal de la pudrición de frutos del mango, por carbendazim (400 ppm), seguido de captan (450 ppm), tiofanato de metilo (450 ppm), ziram (600 ppm) y clorotalonil (650 ppm). Yadav y Majumdar (2004) reportaron la efectividad de carbendazim y mancozeb contra L. theobromae (aislamiento de guayaba). Khanzada et al. (2005) observaron una inhibición significativa del crecimiento de micelio de L. theobromae por carbendazim y tiofanato-metílico cuando se usó a 1 ppm o más de ingrediente activo. Varela et al. (2013) reportaron en cítricos la aplicación de benomilo y compuestos a base de oxicloruro de cobre contra $L$. theobromae en las distintas etapas del cultivo. Li et al. (1995) evaluaron fungicidas contra L. theobromae y Botryosphaeria dothidea causantes de gomosis en duraznos $\mathrm{y}$ albaricoque, $\mathrm{y}$ encontraron que metil-tiofanato inhibió el crecimiento micelial, la germinación de conidios y controló el desarrollo de la enfermedad en árboles de albaricoque. Pereira et al. (2012) evaluaron la sensibilidad de L. theobromae hacia dos grupos de fungicidas, concluyendo que $91.6 \%$ de 120 aislados provenientes de huertos de papaya fueron sensibles a los ingredientes activos del grupo de carbamatos del tipo metil benzimidazol (benomilo y tiabendazol). En el grupo de fungicidas del tipo inhibición por demetilación (imazalil, procloraz, tebuconazol), se encontró gran variabilidad en cuanto al grado de sensibilidad de los aislados analizados; con lo cual se concluyó que L. theobromae es menos sensible a este grupo de fungicidas. Bester et al. (2007) evaluaron diez fungicidas in vitro, para observar la eficacia en la inhibición micelial de las cuatro especies más comunes o patógenas de Botryosphaeriaceae en Sudáfrica: Botryosphaeria obtusa, Neofusicoccum australe, N. parvum y Lasiodiplodia theobromae. Iprodiona, pyrimethanil, acetato de cobre y amonio, kresoxim-metilo y boscalid fueron ineficaces para inhibir el crecimiento micelial a las concentraciones más altas ensayadas $(20 \mu \mathrm{g} / \mathrm{mL}$ para el acetato de cobre y amonio, $5 \mu \mathrm{g}$ / $\mathrm{mL}$ para otros productos evaluados), mientras que benomilo, tebuconazol, cloruro de procloraz manganeso y el flusilazol fueron los fungicidas más efectivos con valores de EC50 para las diferentes especies que oscilaron entre 0.36-0.55, 0.07-0.17, 0.07-1.15 y 0.04-0.36 $\mu \mathrm{g} / \mathrm{mL}$, respectivamente. Syed et al. (2014) llevaron a cabo estudios comparativos de seis fungicidas (carbendazim, Helicoverpa zea single capsid nucleopolyhedrovirus [VPN-HzSNPV], tebuconazole, trifloxystrobin, difenoconazol, tiabendazol y propiconazol), cada uno se evaluó en siete concentraciones ( 1 ppm, 10 ppm, 100 ppm, 1,000 ppm, 10,000 ppm, 20,000 ppm y 30,000 ppm), todos los fungicidas a altas concentraciones resultaron efectivos para inhibir el crecimiento de L. theobromae. En el caso de carbendazim y tiabendazol, resultaron ser casi efectivos en todas las dosis evaluadas en la inhibición del crecimiento micelial, excepto en 1 ppm y 10 ppm.

Aunque in vitro las dosis de los fungicidas evaluados lograron una inhibición completa en el crecimiento micelial de Lasiodiplodia citricola y Lasiodiplodia theobromae, se recomienda hacer pruebas de campo para determinar los productos promisorios que puedan lograr un control satisfactorio de la enfermedad, debido a que bajo estas condiciones intervienen factores que pueden afectar la efectividad del producto y que evitan el contacto con el hongo dentro del hospedante.

\section{Conclusiones}

Lasiodiplodia theobromae fue sensible a las tres dosis evaluadas de los fungicidas Ziram, Carbendazim, Thiram, Tiofanato metílico, Tiabendazol, Cyprodinil + Fludioxonil, Iprodiona, Trichoderma harzianum, Benomilo, con inhibición total del crecimiento micelial del patógeno.

Lasiodiplodia citricola fue sensible a las tres dosis de los fungicidas Ziram, Carbendazim, Thiram, Tiofanato metílico, Tiabendazol, Iprodiona, Trichoderma harzianum, Benomilo y presento crecimiento en las dosis de Cyprodinil + fludioxonil y el testigo absoluto, siendo estadísticamente diferentes.

\section{LITERATURA CITADA}

Abdollahzadeh J, Javadi A, Goltapeh EM, Zare R, Phillips AJL. 2010. Phylogeny and morphology of four new species of Lasiodiplodia from Iran. Persoonia 25: 1-10. https://dx.doi.org/10.3767\%2F003158510X524150

Acosta-Pérez JA, Ortiz-Garcia CF, Zaldívar-Cruz JM, Rodríguez-Cuevas M, Bautista-Muñoz CC, Castillo-Aguilar CdelaC. 2012. Identificación del agente causal e 
importancia de la gomosis en la zona citrícola de Huimanguillo, Tabasco, México. Universidad y Ciencia 28(3): 245-258.

Banik AK, Kaiser SAKM, Dhua RS. 1998. Evaluation of some systemic and non-systemic fungicides against Botryodiplodia theobromae, the cause of dieback disease of mango (Mangifera indica L.). Journal of Soil \& Crops 8: 199-222.

Bester W, Crous PW, Fourie PH. 2007. Evaluation of fungicides as potential grapevine pruning wound protectants against Botryosphaeria species. Australasian Plant Pathology 36(1): 73-77. https:// dx.doi.org/10.1071/AP06086

[CESVMOR] Comité Estatal de Sanidad Vegetal del Estado de Morelos. 2010. Situación actual de la citricultura en el Estado de Morelos. Monitor Agricola 14: 5-13.

Fawcett HS. 1936. Citrus Diseases and Their Control. New York, USA: McGraw-Hill.

Frezzi MJ. 1950. Las especies de Phytophthora en la Argentina. Revista de Investigaciones Agrícolas 4: 47-133.

Khanzada MA, Lodhi AM, Shahzas S. 2005. Chemical control of Lasiodiplodia theobromae the causal agent of mango decline in Sindh. Pakistan Journal of Botany 37: 10231030.

Li H-Y, Cao R-B, Mu Y-T. 1995. In vitro inhibition of Botryosphaeria dothidea and Lasiodiplodia theobromae, and chemical control of gummosis disease of Japanese apricot and peach trees in Zhejiang Province, China. Crop Protection 14(3): 187-191. https://doi.org/10.1016/0261-2194(95)00011-A

Nath K, Solanky KU, Kumawat GL. 2014. Effective approaches of potential bioagent, phytoextract, fungicide and cultural practice for management of banana fruit rot disease. Journal of Plant Pathology \& Microbiology 5: 246. https://doi.org/10.4172/2157-7471.1000246

Pereira AVdaS, Martins RB, Michereff SJ, da Silva MB, Câmara MPS. 2012. Sensitivity of Lasiodiplodia theobromae from Brazilian papaya orchards to MBC and DMI fungicides. European Journal of Plant Pathology 132(4): 489-498. https://doi.org/10.1007/s10658-011-9891-2

Schwentesius RR, Gómez CMA. 2005. Lima 'Persa'. Tendencias en el mercado. Universidad Autónoma Chapingo-Centro de Investigaciones Económicas, Sociales y Tecnológicas de la Agroindustria y la Agricultura Mundial-Programa Integración Agricultura Industrial (PIAI-CIESTAAM). ISBN: 968-02-0167-8. Impreso en México. Imprenta Universitaria UACh. 152 pp.

[SIAP] Servicio de Información Agroalimentaria y Pesquera. 2016. México es $2^{\circ}$ productor mundial de este cítrico. En línea: http://www.gob.mx/siap/articulos/limon-casi-2-44-millones-de-toneladas-en-201 Consultado en línea el 14 de junio de 2017.

Syed RN, Mansha N, Khaskheli MA, Khanzada MA, Lodhi AM. 2014. Chemical control of stem end roto of mango caused by Lasiodiplodia theobromae. Pakistan Journal of Phytopathology 26(2): 201-206.

Tovar JM, Mora JA, Nava C, Téliz D, Villegas Á, Leyva SG. 2013. Control of Lasiodiplodia theobromae, the causal agent of dieback of sapote mamey (Pouteria sapota
(Jacq.) H. E. Moore and Stearn) grafts in Mexico. Revista Fitotecnia Mexicana 36(3): 233-238.

Valle-de la Paz M, Guillén-Sánchez D, Alia-Tejacal I, López-Martínez V, Juárez-López P, Martínez-Fernández E, Hernández-Arenas M, Ariza-Flores R. 2015. Incidencia y severidad de la gomosis (Phytophthora sp.) en lima 'Persa', en Morelos, México. Revista Mexicana de Fitopatología 33(Suplemento): S180.

Valle M. 2018. Muerte descendente (Lasiodiplodia sp. Pat.) en lima Persa (Citrus latifolia Tanaka), en el estado de Morelos. Tesis de doctorado en Ciencias. México: Universidad Autónoma del Estado de Morelos.

Valle-de la Paz M, Guillén-Sánchez D, Guijón-Hernández A, Alia-Tejacal I, López-Martínez V, Juárez-López P, Martínez-Fernández V, Hernández-Arenas M, Ariza-Flores F. 2019. Species of Lasiodiplodia in lima 'Persa' (Citrus latifolia Tanaka) in Morelos, México. Revista BioCiencias 6: e595. https://doi.org/10.15741/revbio.06.01.35

Varela SE, Orozco SM, Torres ARI, Silva AGL. 2013. Guía técnica para la identificación y manejo de plagas y enfermedades en cítricos. Ciudad Victoria, México: Universidad Autónoma de Tamaulipas.

Varela GF. [internet]. 2019. Importancia y distribución de los cítricos. Disponible en: https://tecnoagro.com.mx/revista/2018/no-124/importancia-y-distribucion-de-los-citricos/

Vidales JA. 1982. Etiología de la gomosis de los cítricos en Tecomán, Colima y búsqueda de fuentes de resistencia. Tesis de maestría en Ciencias. México: Universidad Autónoma Chapingo.

Yadav RK, Majumdar VL. 2004. Efficacy of plant extracts, biological agents and fungicides against Lasiodiplodia theobromae incited die back of guava (Psidium guajava). Journal of Mycology and Plant Pathology 34: 415-417. 